\title{
THE INITIAL DISPERSION OF SOLUBLE MATTER IN THREE-DIMENSIONAL FLOW
}

\author{
N. G. BARTON
}

(Received 31 August 1977)

(Revised 13 January 1978)

\begin{abstract}
The dispersion of a passive solute in three-dimensional flow is examined for short times after the injection of solute. If the diffusivity is constant, the solute at first diffuses isotropically about the fluid particle originally coincident with the injection point whilst, at longer times, the effect of diffusion across a velocity shear becomes more important. An asymptotic expansion is derived for the concentration of solute at small times after its injection into the fluid flow and the use of the theory is illustrated for three representative flows. Some critical remarks on the applicability and limitations of the results conclude the note.
\end{abstract}

\section{Introduction}

The first analytic description of the dispersion of soluble material in fluid flow was given by Taylor $[14,15]$ who pointed out that the dispersion relies on diffusion (and convection for three-dimensional flows) across a velocity shear. Taylor examined the dispersion of solute in laminar and turbulent flow in circular pipes and presented a theory which was applicable at asymptotically large times. Most subsequent theoretical investigations into the dispersion mechanism have been concerned principally with asymptotic large-time behaviour. Thus Aris [2] extended Taylor's theory to include longitudinal diffusion, Elder [9] looked at the dispersion of solute in turbulent channel flow, Erdogan and Chatwin [10] examined the influence of small curvature and weak buoyancy forces, and Chatwin [5] and Barton $[3,4]$ described the approach to the asymptotic state for passive and weakly buoyant markers. 
Recently, a few papers have appeared which examine dispersion of solutes at less than asymptotically large times-for example, Sullivan [13] and Chatwin [6] have looked at dispersing solutes in turbulent flow at moderate to large times. There remain comparatively few results for asymptotically short times, however, and those that exist appear to be only for uni-directional flow. Thus Lighthill [11] examined the onset of dispersion in Poiseuille flow and his results were extended by Chatwin [7,8] using an earlier suggestion by Saffman [12].

It seems worth while, therefore, to present a theory for the dispersion of solutes in general three-dimensional flows at asymptotically short times. The analysis in this paper will be restricted to considering passive solutes (that is, solutes which do not give rise to dynamical forces) which have a constant diffusivity $\kappa$. The theory would be expected to have most application to biological or chemical processes in which solutes might be dispersed in laminar flows; it would not, however, have much relevance to oceanographical or meteorological problems in which the concept of an eddy diffusivity would have to apply. Eddy diffusivities represent effects observed after averaging the characteristics of the flow and it seems unreasonable to apply an asymptotic short-time limit to such an averaged representation. The theory would, in any case, require substantial modification for such problems as eddy diffusivities are generally not modelled as constants.

The bulk of this paper considers the dispersal of solutes for times less than that required for solute particles to diffuse across the domain, that is, $t<O\left(L^{2} / \kappa\right)$ where $L$ is a typical length scale and $\kappa$ is the diffusivity. This means that solutes injected into fluid flow away from boundaries will not have time to "feel" the presence of boundaries and it enables a simplified boundary condition to be taken, namely $C \rightarrow 0$ far from the injection point instead of $\partial C / \partial n=0$ at the boundary. Chatwin [8] has presented some results for the initial dispersion of solute close to the boundary in Poiseuille flow and the present paper does, in one case, consider the effect of a nearby plane boundary (Section 4). However, a general theory in which the boundary condition $\partial C / \partial n=0$ at given boundaries is satisfied requires numerical rather than analytic methods.

At short times $t<O\left(L^{2} / \kappa\right)$, diffusion will spread injected solute through a distance of $O\left((\kappa t)^{\frac{1}{2}}\right)$ whilst diffusion across a velocity shear (that is, the dispersion mechanism) spreads solute over a distance of order $(\kappa t)^{\frac{1}{2}} \times$ velocity gradient $\times t$. It is clear that diffusion, acting like $t^{\frac{1}{2}}$, will dominate the dispersion mechanism for very short times whilst the two effects would balance for times nominally of order (velocity gradient) ${ }^{-1}$. Here "velocity gradient" implies the maximum velocity gradient, for example, across the cross-section in Poiseuille flow. Thus solute injected into fluid flow will initially diffuse isotropically about the moving fluid particle which originally coincided with the point of injection of the solute. This fluid particle will have moved only a small distance during the isotropic diffusion stage. The 
dispersion mechanism will come into play at slightly larger times and the aim of this note is to describe the consequent development of anisotropy in the cloud of solute.

In Section 2, the isotropic diffusion about the moving point will be described and the development of anisotropy will be investigated using an asymptotic expansion for the concentration. In Section 3, equations for the coefficients in the asymptotic series will be solved to obtain the leading four terms. Higher terms could be obtained but it is argued that four terms are sufficient in the expansion. In Section 4, the initial dispersion of solute in three representative flows (one of which includes the presence of a plane boundary) will be considered and some critical remarks on the results will conclude the note.

\section{An asymptotic expansion for the concentration at short times}

Suppose that a solute with constant diffusivity $\kappa$ is injected into a threedimensional flow at $\mathbf{r}_{0}$ at time $t_{0}$, so that the concentration $C$ of the solute satisfies

$$
\frac{\partial C}{\partial t}+\mathbf{u} \cdot \nabla C=\kappa \nabla^{2} C
$$

with

$$
C=\delta\left(\mathbf{r}-\mathrm{r}_{0}\right) \quad \text { at } t=t_{0} .
$$

For very small times, the cloud of solute spreads isotropically about the fluid particle initially coincident with the injection point (Saffman [12], Chatwin [7]) so that the concentration is approximately

$$
C(\mathbf{r}, t)=\frac{A}{8\left[\pi \kappa\left(t-t_{0}\right)\right]^{\mathrm{p}}} \exp \{-\mathbf{R} \cdot \mathbf{R}\},
$$

where $A$ is a constant which fixes the total amount of solute and $R$ is a nondimensional similarity variable,

$$
\mathbf{R}=\left\{\mathbf{r}-\mathbf{r}_{0}-\left(t-t_{0}\right) \mathbf{u}\left(\mathbf{r}_{0}\right)\right\} / 2\left[\kappa\left(t-t_{0}\right)\right]^{\mathbf{z}} .
$$

For slightly larger times when the dispersion mechanism begins to operate, a formal solution to $(2.1,2.2)$ will be given by a series expansion which has $(2.3)$ as its leading term. The most concise series (found by Chatwin [7]) takes the form

$$
C=\frac{A}{8 \pi \kappa\left(t-t_{0}\right)} \exp \{-\mathbf{R} \cdot \mathbf{R}\} \sum_{n=0}^{\infty} G_{n}(\mathbf{R}) T^{n}, \quad G_{0}(\mathbf{R})=1,
$$

where $T$ is the non-dimensional variable

$$
T=\left(\kappa\left(t-t_{0}\right) / L^{2}\right)^{\frac{1}{2}}
$$


in which $L$ is a characteristic length of the phenomenon being investigated. Equations for the coefficient functions $G_{n}(\mathbf{R}), n \geqslant 1$, in (2.5) follow by substituting (2.5) in (2.1) and equating the coefficients of powers of $T$ to zero. Naturally the solutions $G_{n}(\mathbf{R})$ must be finite as $|\mathbf{R}| \rightarrow 0$ and, recalling the assumption in Section 1, they also should have the property

$$
G_{n}(\mathbf{R}) \exp \{-\mathbf{R} \cdot \mathbf{R}\} \rightarrow 0 \text { as }|\mathbf{R}| \rightarrow \infty
$$

if the effects of boundaries are to be ignored.

The coefficient functions $G_{1}(\mathbf{R})$ to $G_{4}(\mathbf{R})$ have been found by Chatwin [7] for Poiseuille flow and will now be obtained for general three-dimensional flow away from boundaries.

For very small times, it is useful to group the dominant terms of equation (2.1) as

$$
\frac{\partial C}{\partial t}+\mathbf{u}\left(\mathbf{r}_{0}\right) \cdot \nabla C-\kappa \nabla^{2} C=-\left\{\mathbf{u}(\mathbf{r})-\mathbf{u}\left(r_{0}\right)\right\} \cdot \nabla C
$$

in which the right-hand side is presumed to be small near the injection point. Using Taylor's series for a vector valued function of three variables it is possible and convenient to write

$$
\begin{aligned}
\mathbf{u}(\mathbf{r})-\mathbf{u}\left(\mathbf{r}_{0}\right)=\left\{\left(x_{j}-\right.\right. & \left.x_{j}^{0}\right) u_{i, j}\left(\mathbf{r}_{0}\right)+\frac{1}{2 !}\left(x_{j}-x_{j}^{0}\right)\left(x_{k}-x_{k}^{0}\right) u_{i, j k}\left(\mathbf{r}_{0}\right) \\
& \left.+\frac{1}{3 !}\left(x_{j}-x_{j}^{0}\right)\left(x_{k}-x_{k}^{0}\right)\left(x_{l}-x_{l}^{0}\right) u_{i, j k l}\left(\mathbf{r}_{0}\right)+\ldots\right\} \mathbf{e}_{i}
\end{aligned}
$$

in which the summation convention is used, the $\mathbf{e}_{i}$ are an orthonormal triad, $\mathbf{r}=x_{i} \mathbf{e}_{i}, \mathbf{r}_{0}=x_{i}^{0} \mathbf{e}_{i}$ and the comma denotes partial differentiation, for example,

$$
u_{i, j k}\left(\mathbf{r}_{0}\right)=\left.\frac{\partial^{2} u_{i}}{\partial x_{j} \partial x_{k}}\right|_{\mathbf{r}=\mathbf{r}_{0}}
$$

Now using equations $(2.4,2.6)$ it follows that

$$
\mathbf{R}=X_{i} \mathbf{e}_{i}=\frac{1}{2 L T}\left(\mathbf{r}-\mathbf{r}_{0}\right)-\frac{L T}{2 \kappa} \mathbf{u}\left(\mathbf{r}_{0}\right)
$$

or

$$
\mathbf{r}-\mathbf{r}_{0}=\left(x_{i}-x_{i}^{0}\right) \mathbf{e}_{i}=2 L T \mathbf{R}+\frac{L^{2} T^{2}}{\kappa} \mathbf{u}\left(\mathbf{r}_{0}\right) .
$$

Finally, equations $(2.6,2.10)$ give the transformations

$$
\frac{\partial}{\partial t} \rightarrow\left(\frac{\partial}{\partial T}+\frac{\partial}{\partial X_{i}} \frac{d X_{i}}{d T}\right) \frac{d T}{d t}=\frac{\kappa}{2 L^{2} T}\left\{\frac{\partial}{\partial T}-\frac{1}{T} \mathbf{R} \cdot \nabla^{\prime}-\frac{L}{\kappa} \mathbf{u}\left(\mathbf{r}_{0}\right) \cdot \nabla^{\prime}\right\}
$$


and

$$
\nabla \rightarrow \frac{1}{2 L T} \nabla^{\prime}
$$

where $\nabla^{\prime}=\mathbf{e}_{i}\left(\partial / \partial X_{i}\right)$, whence an equation that enables the determination of the $G_{n}(\mathbf{R})$ is obtained by substituting the series (2.5) in (2.8). After some simplification the result is

$$
\begin{aligned}
& \frac{\kappa}{2 L^{2} T} \sum_{n=0}^{\infty} G_{n}(\mathbf{R})(n-3) T^{n-4}-\frac{\kappa}{2 L^{2} T^{2}} \mathbf{R} \cdot\left[\sum_{n=0}^{\infty}\left(-2 \mathbf{R} G_{n}(\mathbf{R})+\nabla^{\prime} G_{n}\right) T^{n-3}\right] \\
& -\frac{\kappa}{4 L^{2} T^{2}} \sum_{n=0}^{\infty}\left\{\left(4|\mathbf{R}|^{2}-6\right) G_{n}-4 \mathbf{R} \cdot \nabla^{\prime} G_{n}+\nabla^{\prime 2} G_{n}\right\} T^{n-3} \\
& =\frac{1}{2 L T}\left\{\left(2 L T X_{j}+\frac{L^{2} T^{2}}{\kappa} u_{j}\left(\mathbf{r}_{0}\right)\right) u_{i, j}\left(\mathbf{r}_{0}\right)+\frac{1}{2 !}\left(2 L T X_{j}+\frac{L^{2} T^{2}}{\kappa} u_{j}\left(\mathbf{r}_{0}\right)\right)\right. \\
& \quad \times\left(2 L T X_{k}+\frac{L^{2} T^{2}}{\kappa} u_{k}\left(\mathbf{r}_{0}\right)\right) u_{i, j k}\left(\mathbf{r}_{0}\right)+\frac{1}{3 !}\left(2 L T X_{j}+\frac{L^{2} T^{2}}{\kappa} u_{j}\left(\mathbf{r}_{0}\right)\right) \\
& \left.\quad \times\left(2 L T X_{k}+\frac{L^{2} T^{2}}{\kappa} u_{k}\left(\mathbf{r}_{0}\right)\right)\left(2 L T X_{l}+\frac{L^{2} T^{2}}{\kappa} u_{l}\left(\mathbf{r}_{0}\right)\right) u_{i, j k l}\left(\mathbf{r}_{0}\right)+\ldots\right\} \\
& \quad \times\left\{\sum_{n=0}^{\infty}\left(2 X_{i} G_{n}(\mathbf{R})-\frac{\partial G_{n}}{\partial X_{i}}\right) T^{n-3}\right\}
\end{aligned}
$$

in which the right-hand side follows from equations $(2.9,2.10,2.11)$.

The following equations for the coefficient functions $G_{n}(\mathbf{R})(n=1, \ldots, 4)$ are obtained by equating the coefficients of $T^{m}$ to zero $(m=-4,-3,-2,-1)$ in (2.13):

$$
\begin{aligned}
\frac{\kappa}{L^{2}}\left\{\frac{1}{2} G_{1}(\mathbf{R})+\frac{1}{2} \mathbf{R} \cdot \nabla^{\prime} G_{1}-\frac{1}{4} \nabla^{\prime 2} G_{1}\right\} & =0 \\
\frac{\kappa}{L^{2}}\left\{G_{2}(\mathbf{R})+\frac{1}{2} \mathbf{R} \cdot \nabla^{\prime} G_{2}-\frac{1}{4} \nabla^{\prime 2} G_{2}\right\} & =A_{i} 2 X_{i}, \\
\frac{\kappa}{L^{2}}\left\{\frac{3}{2} G_{3}(\mathbf{R})+\frac{1}{2} \mathbf{R} \cdot \nabla^{\prime} G_{3}-\frac{1}{4} \nabla^{\prime 2} G_{3}\right\} & =B_{i} 2 X_{i}+A_{i}\left(2 X_{i} G_{1}(\mathbf{R})-\frac{\partial G_{1}}{\partial X_{i}}\right), \\
\frac{\kappa}{L^{2}}\left\{2 G_{4}(\mathbf{R})+\frac{1}{2} \mathbf{R} \cdot \nabla^{\prime} G_{4}-\frac{1}{4} \nabla^{\prime 2} G_{4}\right\} & =C_{i} 2 X_{i}+B_{i}\left(2 X_{i} G_{1}(\mathbf{R})-\frac{\partial G_{1}}{\partial X_{i}}\right) \\
& +A_{i}\left(2 X_{i} G_{2}(\mathbf{R})-\frac{\partial G_{2}}{\partial X_{i}}\right) .
\end{aligned}
$$


The terms $A_{i}, B_{i}, C_{i}$ on the right-hand side are found to be

$$
\begin{aligned}
A_{i} & =X_{j} u_{i, j}\left(\mathbf{r}_{0}\right), \\
B_{i} & =\frac{L}{2 \kappa} u_{j}\left(\mathbf{r}_{0}\right) u_{i, j}\left(\mathbf{r}_{0}\right)+L X_{j} X_{k} u_{i, j k}\left(\mathbf{r}_{0}\right), \\
C_{i}= & \frac{L}{2 \kappa}\left(X_{j} u_{k}\left(\mathbf{r}_{0}\right)+u_{j}\left(\mathbf{r}_{0}\right) X_{k}\right) u_{i, j k}\left(\mathbf{r}_{0}\right) \\
& +\frac{1}{3 !}(2 L)^{2} X_{j} X_{k} X_{l} u_{i, j k l}\left(\mathbf{r}_{0}\right) .
\end{aligned}
$$

There are other possible short time expansions for the concentration (mainly based on variations in the exponent in (2.5)), but the one above seems to be the most concise. Equations for the higher coefficient functions $G_{5}(\mathbf{R}), G_{6}(\mathbf{R}), \ldots$ could be obtained readily enough but this hardly seems necessary for two reasons. Firstly, the algebra involved in the derivation and use gets progressively more cumbersome and, secondly (and more importantly), the series (2.5) is expected to be asymptotic as $t \rightarrow 0$ and typically such series diverge as more terms are taken. That is, for a given small value of $t$, the error in the series (2.5) would ultimately be increased by including more terms in the series. For both reasons, there seems no point in extending the series beyond four terms.

\section{Solutions for the coefficient functions}

The equations $(2.14 a-d, 2.15 a-c)$ derived in the previous section are now solved for the coefficient functions $G_{n}(\mathbf{R})$. Firstly, it is observed that the equation for each $G_{n}(\mathbf{R})$ has the confluent hypergeometric functions $M\left(\frac{1}{2} n, \frac{3}{2}, R^{2}\right)$ and $U\left(\frac{1}{2} n, \frac{3}{2}, R^{2}\right)$ as complementary functions, $(R=|\mathbf{R}|)$. Both these solutions must be neglected since

$$
M\left(\frac{1}{2} n, \frac{3}{2}, R^{2}\right) \exp \left(-R^{2}\right) \sim \Gamma\left(\frac{3}{2}\right) R^{n-3} / \Gamma\left(\frac{1}{2} n\right) \quad \text { as } R \rightarrow \infty \text { * }
$$

and, bearing (2.7) in mind, this would lead to an infinite amount of solute once a volume integral were to be taken. Also

$$
U\left(\frac{1}{2} n, \frac{3}{2}, R^{2}\right) \sim \sqrt{ }(\pi) / \Gamma\left(\frac{1}{2} n\right) R \text { as } R \rightarrow 0 *
$$

which would lead to an infinite concentration at $R=0$ if $U\left(\frac{1}{2} n, \frac{3}{2}, R^{2}\right)$ were not neglected.

\footnotetext{
* See, for example, Abramowitz and Stegun ([1, p. 508]).
} 
The particular solutions of equations $(2.14 \mathrm{a}-\mathrm{d}, 2.15 \mathrm{a}-\mathrm{c})$ are polynomials in $X_{i}$ and have the form

$$
\begin{aligned}
& G_{1}(\mathbf{R})=0, \\
& G_{2}(\mathbf{R})=\alpha+\beta_{i j} X_{i} X_{j}, \\
& G_{3}(\mathbf{R})=\gamma_{i} X_{i}+\Delta_{i j k} X_{i} X_{j} X_{k}, \\
& G_{4}(\mathbf{R})=\varepsilon+\zeta_{i j} X_{i} X_{j}+\eta_{i j k l} X_{i} X_{j} X_{k} X_{l}
\end{aligned}
$$

in which the coefficients are found to be

$$
\begin{aligned}
\alpha & =\frac{1}{2} \beta_{i i}, \\
\beta_{i j} & =\frac{L^{2}}{\kappa} u_{i, j}\left(\mathbf{r}_{0}\right), \\
\gamma_{i} & =\frac{1}{2}\left\{\frac{L^{3}}{\kappa^{2}} u_{j}\left(\mathbf{r}_{0}\right) u_{i, j}\left(\mathbf{r}_{0}\right)+\frac{1}{2}\left(\Delta_{i j j}+\Delta_{j i j}+\Delta_{j j i}\right)\right\}, \\
\Delta_{i j k} & =\frac{2}{3} \frac{L^{3}}{\kappa} u_{i, j k}\left(\mathbf{r}_{0}\right), \\
\varepsilon & \left.=\frac{1}{4} \zeta_{i i}, \quad+\frac{L^{2}}{\kappa}\left(2 \alpha u_{i, j}\left(\mathbf{r}_{0}\right)-\left(\beta_{k i}+\beta_{i k}\right) u_{k, j}\left(\mathbf{r}_{0}\right)\right)\right\}, \\
\zeta_{i j} & =\frac{1}{3}\left\{\frac{L^{4}}{\kappa^{2}} u_{k}\left(\mathbf{r}_{0}\right)\left(u_{i}, j\left(\mathbf{r}_{0}\right)+u_{i, k j}\left(\mathbf{r}_{0}\right)\right)\right. \\
\eta_{i j k l} & =\frac{1}{4}\left\{\frac{4 L^{4}}{3 \kappa} u_{i, j k l}\left(\mathbf{r}_{0}\right)+\frac{2 L^{2}}{\kappa} \beta_{k l} u_{i, j}\left(\mathbf{r}_{0}\right)\right\} .
\end{aligned}
$$

\section{Applications}

Perhaps the most important application of the analysis is to describe the initial dispersion of solute prior to using a numerical solution. Dispersion from an initial point source would pose difficulties for a finite difference scheme because a very fine mesh of computation points would be required for reasonable numerical accuracy. The short-time analysis would describe the initial dispersion from a point source up to a time when relatively coarse meshes could give reasonable accuracy. The implementation of the short-time analysis would be the analogue of "extracting the singularity" in numerical integration.

The analysis is almost as easy to apply to three-dimensional flows as to twodimensional flows. This contrasts with the direct numerical solution of the 
convective-diffusive equation (2.1) in which numerical schemes for threedimensional flows are significantly more complicated than those for two-dimensional flows. The results shown in this section are for two-dimensional flows merely for ease of presentation.

One instance in which the above theory may be applied has already been examined by Chatwin [7]. Chatwin's results were for the initial dispersion of solute in Poiseuille flow representing a model of dispersion of tracers in blood flow in the aorta. The theory is applied to another three common cases below, namely initial dispersal of solutes in flow near a stagnation point, in flow near a point vortex and in plane Poiseuille flow. The dispersion of solutes in these sorts of flows is doubtless an everyday experience; for example, the plane Poiseuille flow case might be used to examine the initial mixing of a contaminant in a rectangular air-conditioning duct. Perhaps the best general application of the theory might be for contaminant gases starting to disperse in gaseous flow as, in such cases, laminar flows may still be common enough and the diffusivity $\kappa$ is large enough (typically 0.1 to $1 \mathrm{~cm}^{2} \mathrm{~s}^{-1}$ ) for diffusion to be observable and important over reasonably short times.

The third example discussed in this section is a case of initial dispersion near a plane boundary where the condition $\partial C / \partial n=0$ has to apply. This condition must be applied whenever the solute has had time to diffuse near to solid boundaries and the condition is satisfied by considering dispersion in an additional image flow field. Of course, an image flow field becomes difficult to construct if the boundary is anything other than very simple and, in general, the presence of nearby boundaries would mean a full numerical solution is required.

\section{Initial dispersion near a stagnation point}

Consider a flow near a stagnation point at $x=y=0$; thus take as velocity components

$$
u_{1}=-U x / L, \quad u_{2}=U y / L, \quad u_{3}=0
$$

and suppose that solute is injected at $t=t_{0}$ at $\mathbf{r}_{0}=x_{0} \mathbf{e}_{1}+y_{0} \mathbf{e}_{2}+z_{0} \mathbf{e}_{3}$. The series (2.5) for the concentration is found to take the form

$$
C=\frac{A}{8\left(\pi \kappa\left(t-t_{0}\right)\right)^{\frac{1}{4}}} \exp \{-\mathbf{R} \cdot \mathbf{R}\}\left\{1-\frac{L U}{\kappa}\left(X_{1}^{2}-X_{2}^{2}\right) T^{2}+\frac{L U^{2}}{2 \kappa^{2}}\left(x_{0} X_{1}+y_{0} X_{2}\right) T^{3}+0\left(T^{4}\right)\right\}
$$

where $X_{1}, X_{2}, X_{3}$ are given by

$$
X_{1}=\frac{x-x_{0}+\left(t-t_{0}\right) U x_{0} / L}{2\left(\kappa\left(t-t_{0}\right)\right)^{\frac{1}{2}}}, \quad X_{2}=\frac{y-y_{0}-\left(t-t_{0}\right) U y_{0} / L}{2\left(\kappa\left(t-t_{0}\right)\right)^{\frac{1}{2}}}, \quad X_{3}=\frac{z-z_{0}}{2\left(\kappa\left(t-t_{0}\right)\right)^{\frac{1}{z}}} .
$$


Thus the concentration develops a skewed Gaussian distribution about the fluid point originally coincident with the injection point. The result (4.2) has been illustrated for a particular set of flow parameters in Fig. 1 and, based on the computations, the theory seems to give reasonable answers provided $t<0.2 L / U$ (see caption and Section 5). The theory does not apply for greater values of $t$ when the curvature of the streamline through the injection point cannot be neglected.

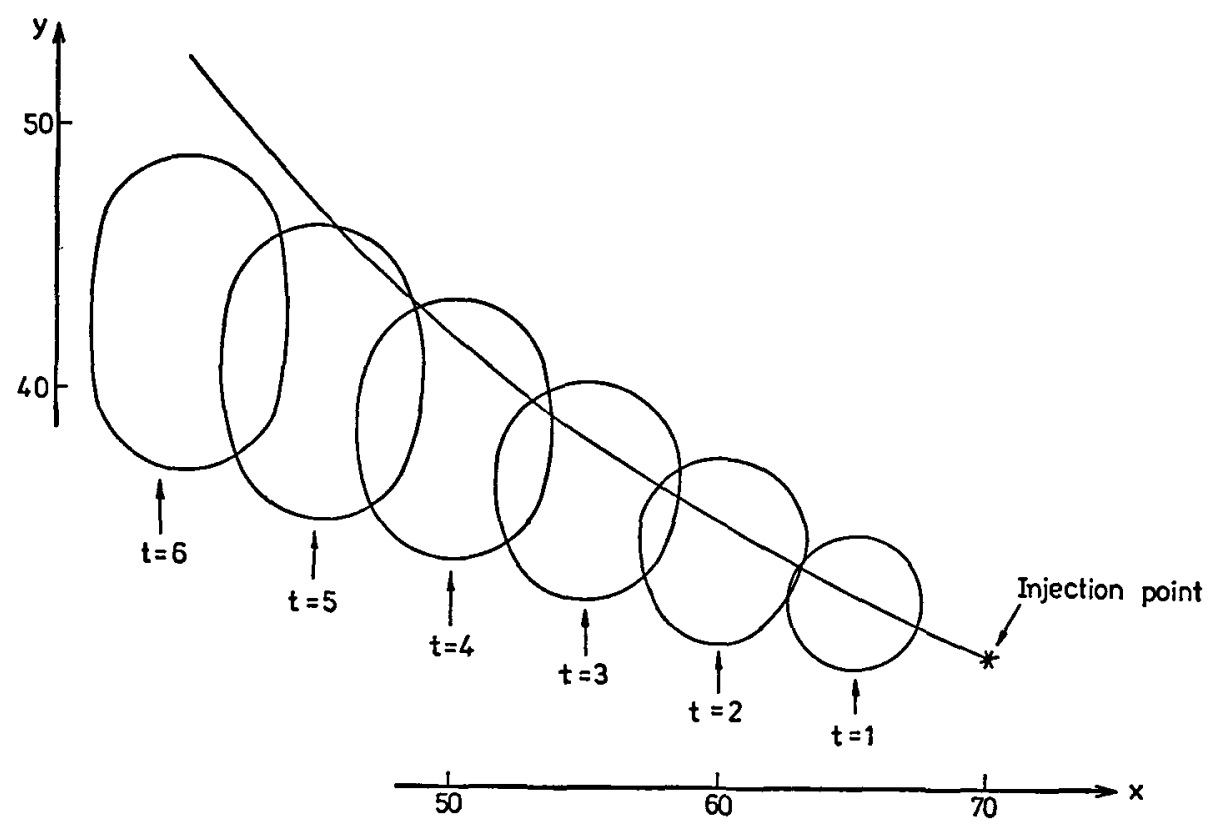

Fig. 1. Initial dispersion from a unit point source near a stagnation point at $x=y=0$; $\kappa=0.2 \mathrm{~cm}^{2} \mathrm{~s}^{-1}, u=-x / 14 \mathrm{~cm} \mathrm{~s}^{-1}, v=y / 14 \mathrm{~cm} \mathrm{~s}^{-1}, x_{0}=70 \mathrm{~cm}, y_{0}=30 \mathrm{~cm}$. The diffusivity is appropriate for the initial dispersion of a gas in air flow. The interior of the curves represent points where $C>10^{-4}$ and the line represents the streamline through the injection point. The effect of streamline curvature is not important for 3 or 4 seconds after injection, corresponding to $t-t_{0}<0.2 L / U$ taking $L=100 \mathrm{~cm}$ and $U=5 \mathrm{~cm} \mathrm{~s}^{-1}$. Three term and four term series gave similar concentration curves.

\section{Initial dispersion near a point vortex}

Next consider a flow near a point vortex at $x=y=0$; the velocity components are therefore given by

$$
u_{1}=\frac{-\alpha y}{x^{2}+y^{2}}, \quad u_{2}=\frac{\alpha x}{x^{2}+y^{2}}, \quad u_{3}=0 .
$$

For this flow the coefficients $G_{1}(\mathbf{R}), \ldots, G_{4}(\mathbf{R})$ given by $(3.1,3.2)$ prove to be very complicated and, consequently, the analytic form of the series $(2.5)$ is omitted here. 
The computed results of a three-term series for the concentration are shown in Fig. 2 for flow near a specified vortex. Again the concentration takes a skewed Gaussian form and the results are clearly inapplicable when the curvature of the streamline through the injection point is important. In this case the theory should give reasonable answers for times after injection less than $0.15 L / U$ (see caption and Section 5).
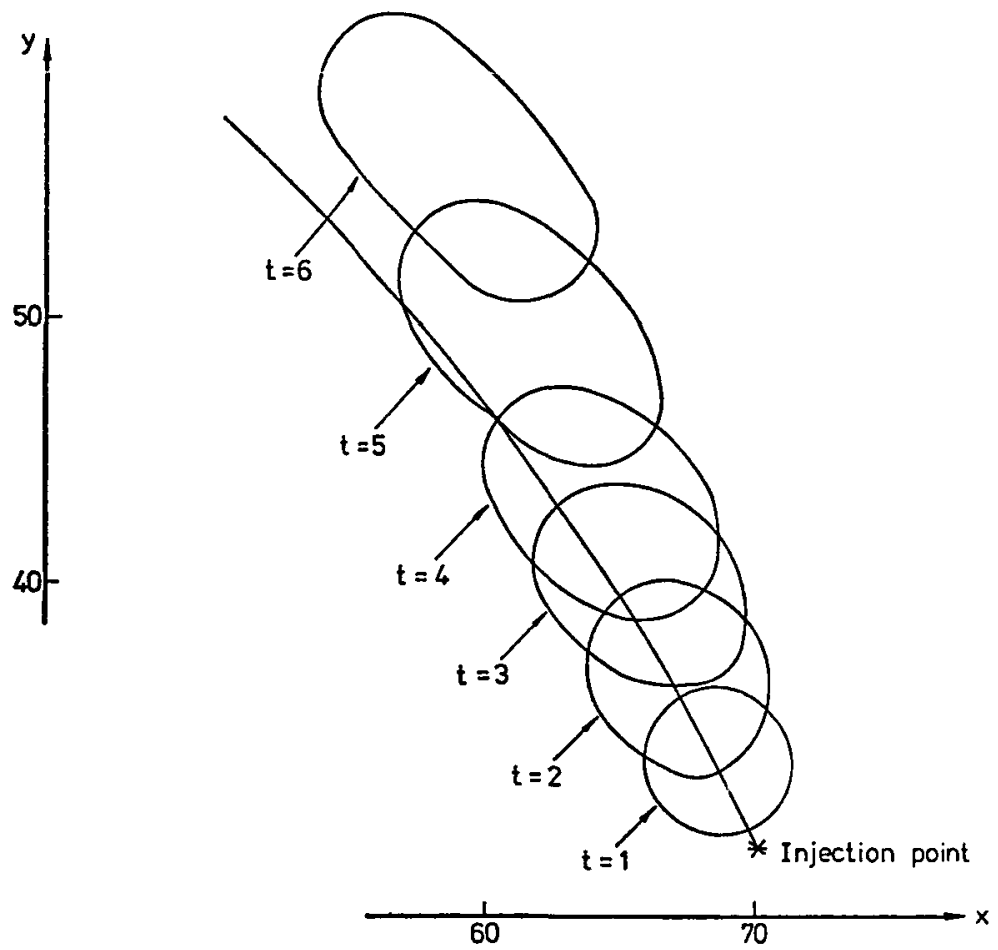

Fig. 2. Initial dispersion from a unit point source near a point vortex at $x=y=0$; $\kappa=0.2 \mathrm{~cm}^{2} \mathrm{~s}^{-1}, \quad u=-\alpha y / x^{2}+y^{2} \mathrm{~cm} \mathrm{~s}^{-1}, \quad v=\alpha x / x^{2}+y^{2} \mathrm{~cm} \mathrm{~s}^{-1}, \quad \alpha \approx 271.5, \quad x_{0}=70 \mathrm{~cm}$, $y_{0}=30 \mathrm{~cm}$. The diffusivity is appropriate for the initial dispersion of a gas in air flow. The interior of the curves represents points where $C>10^{-5}$ and the line represents the streamline through the injection point. The effect of streamline curvature is not important for 4 or 5 seconds after injection, corresponding to $t-t_{0}<0.15 L / U$ taking $L=100 \mathrm{~cm}$ and $U=3 \mathrm{~cm} \mathrm{~s}^{-1}$. The results shown are for a three term series and those for a four term series were unsatisfactory for $t>3 \mathrm{~s}$ when condition (5.3) had been violated. Even so, $t=3 \mathrm{~s}$ corresponds to $t \approx 135 \mathrm{~K} / U^{2}$ showing that $(5.5)$ is quite pessimistic.

\section{Initial dispersion in plane Poiseuille flow}

Plane Poiseuille flow is a particular example of a uni-directional shear flow with velocity components

$$
u_{1}=u(y), \quad u_{2}=u_{3}=0 .
$$


If a solute were to be injected at $t=t_{0}$ at $r_{0}=x_{0} \mathrm{e}_{1}+y_{0} \mathrm{e}_{2}+z_{0} \mathrm{e}_{3}$ into such a flow, the series $(2.5)$ for the concentration takes the form

$$
C=\frac{A}{8\left(\pi \kappa\left(t-t_{0}\right)\right)^{4}} \exp \{-\mathbf{R} \cdot \mathbf{R}\}\left\{1+T^{2} \beta_{12} X_{1} X_{2}+T^{3}\left(\gamma_{1} X_{1}+\Delta_{122} X_{1} X_{2}^{2}\right)+O\left(T^{4}\right)\right\},
$$

where the constants are found to be

$$
\beta_{12}=\frac{L^{2}}{\kappa} u^{\prime}\left(y_{0}\right), \quad \gamma_{1}=\frac{L^{3}}{6 \kappa} u^{\prime \prime}\left(y_{0}\right), \quad \Delta_{122}=\frac{2 L^{3}}{3 \kappa} u^{\prime \prime}\left(y_{0}\right)
$$

and $X_{1}, X_{2}, X_{3}$ are given by

$$
X_{1}=\frac{x-x_{0}-\left(t-t_{0}\right) u\left(y_{0}\right)}{2\left(\kappa\left(t-t_{0}\right)\right)^{\frac{t}{t}}}, \quad X_{2}=\frac{y-y_{0}}{2\left(\kappa\left(t-t_{0}\right)\right)^{\frac{1}{2}}}, \quad X_{3}=\frac{z-z_{0}}{2\left(\kappa\left(t-t_{0}\right)\right)^{\frac{t}{t}}} .
$$

The result (4.6) holds for initial dispersion in unidirectional shear flow far away from boundaries. The concentration develops a skewed Gaussian distribution about the fluid point originally coincident with the injection point and the main features of the skewness are determined by the sign of $\beta_{12}$, that is of $u^{\prime}\left(y_{0}\right)$.

The series (4.6) requires modification when the solute is injected close to a boundary for then the condition $\partial C / \partial n=0$ has to apply at the boundary. This condition may be satisfied by using an additional image flow on the other side of the boundary. The construction of an image flow field is generally only possible for very simple boundaries such as planes and perhaps intersecting planes. By way of example, suppose a solute is injected at $\mathbf{r}_{0}=x_{0} \mathbf{e}_{1}+y_{0} \mathbf{e}_{2}+z_{0} \mathbf{e}_{3}$ close to the plane boundary $y=0$ in the unidirectional shear flow specified by (4.5). The augmented asymptotic expansion for the concentration is then found to have the form

$$
\begin{gathered}
C=\frac{A}{8\left(\pi \kappa\left(t-t_{0}\right)\right)^{4}}\left[\exp \{-\mathbf{R} \cdot \mathbf{R}\}\left\{1+T^{2} \beta_{12} X_{1} X_{2}+T^{3}\left(\gamma_{1} X_{1}+\Delta_{122} X_{1} X_{2}^{2}\right)+O\left(T^{4}\right)\right\}\right. \\
\left.+\exp \{-\mathrm{S} \cdot \mathrm{S}\}\left\{1+T^{2} \tilde{\beta}_{12} Y_{1} Y_{2}+T^{3}\left(\tilde{\gamma}_{1} Y_{1}+\tilde{\Delta}_{122} Y_{1} Y_{2}^{2}\right)+O\left(T^{4}\right)\right\}\right]
\end{gathered}
$$

in which the additional terms are defined by

$$
\begin{array}{cl}
\tilde{\beta}_{12}=-\beta_{12}, & \tilde{\gamma}_{1}=\gamma_{1}, \quad \tilde{\Delta}_{122}=\Delta_{122}, \\
& \mathbf{S}=Y_{i} \mathbf{e}_{i}, \\
Y_{1}=\frac{x-x_{0}-\left(t-t_{0}\right) u\left(y_{0}\right)}{2\left(\kappa\left(t-t_{0}\right)\right)^{\frac{1}{2}}}, & Y_{2}=\frac{y+y_{0}}{2\left(\kappa\left(t-t_{0}\right)\right)^{\frac{1}{3}}}, \quad Y_{3}=\frac{z-z_{0}}{\left.2 \kappa\left(t-t_{0}\right)\right)^{\frac{3}{3}}} .
\end{array}
$$


The series (4.9) was computed for the concentration of a solute close to a boundary in the case of plane Poiseuille flow, that is, where the velocity components are

$$
u_{1}=c_{1} y\left(c_{2}-y\right), \quad u_{2}=u_{3}=0,
$$

and the results are illustrated in Fig. 3.

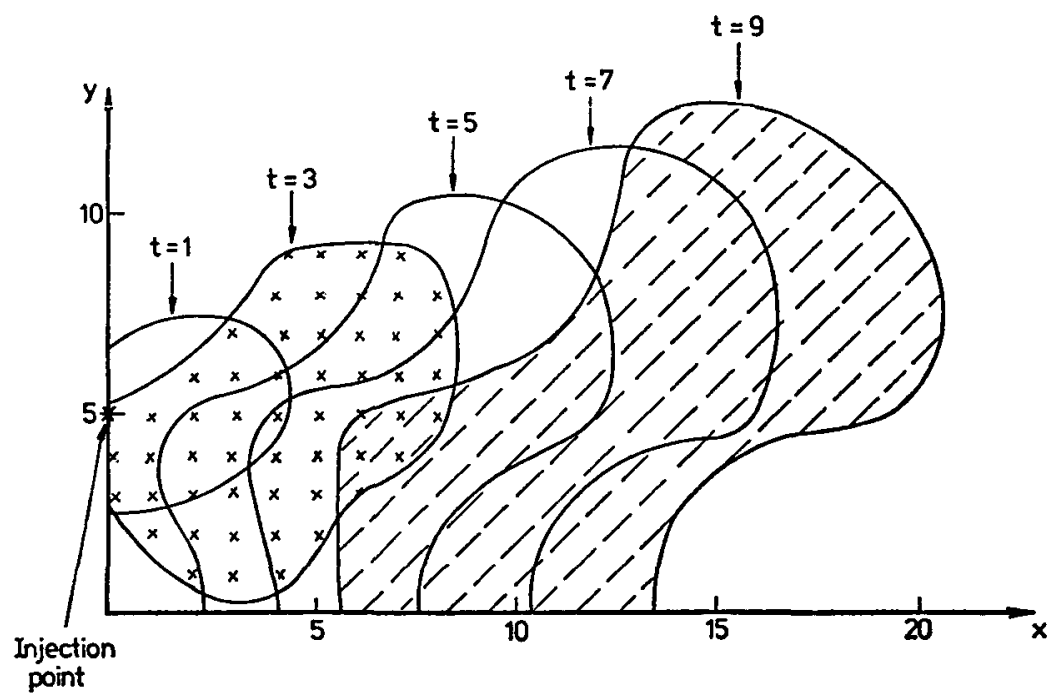

Fig. 3. Initial dispersion from a unit point source close to the boundary in plane Poiseuille flow; $\kappa=0.2 \mathrm{~cm}^{2} \mathrm{~s}^{-1}, u=0.003 y(100-y) \mathrm{cm} \mathrm{s}^{-1}, v=0 \mathrm{~cm} \mathrm{~s}^{-1}, x_{0}=0 \mathrm{~cm}, y_{0}=5 \mathrm{~cm}$. The diffusivity is appropriate for the initial dispersion of a gas in air flow. The interior of the curves represent points where $C>10^{-5}$ and the results have been computed using an additional image field as shown in equation (4.9). Three and four term series gave similar concentration curves for up to three seconds after injection corresponding to $t \approx 30 \kappa U^{2}$. The results shown are for a three term series.

It is difficult to assess the range of applicability of the series (4.9) in this caseand in similar cases when the streamlines are straight. Some critical comments on the source of errors in the asymptotic expansion for the concentration are given in Section 5.

\section{The applicability and limitations of the asymptotic expansion}

The theory presented above can be modified to describe the results of continuous injection of a cloud of solute into a flow. Thus if $F\left(r_{0}, t_{0}\right)\left|d r_{0}\right| d t_{0}$ is the amount of contaminant injected in a volume $\left|\mathbf{d r}_{0}\right|$ at $\mathbf{r}_{0}$ and time $d t_{0}$ at $t_{0}$, the resulting concentration will be given by

$$
C=\iiint_{\text {all space }}\left\{\int_{-\infty}^{t} \frac{F\left(\mathbf{r}_{0}, t_{0}\right)}{8\left(\pi \kappa\left(t-t_{0}\right)\right)^{\frac{1}{2}}} \exp (-\mathbf{R} \cdot \mathbf{R}) \sum_{n=0}^{\infty} G_{n}(\mathbf{R}) T^{n} d t_{0}\right\} \mathbf{d r}_{0},
$$


where

$$
\mathbf{R}=\left\{\mathbf{r}-\mathbf{r}_{0}-\left(t-t_{0}\right) u\left(\mathbf{r}_{0}\right)\right\} / 2\left(\kappa\left(t-t_{0}\right)\right)^{ \pm}, \quad T=\left(\kappa\left(t-t_{0}\right)\right)^{ \pm} / L
$$

and the first four coefficient functions were obtained in Section 3. The volume integration is taken over all space after assuming that the solute has had insufficient time to diffuse to near any boundaries of the flow. The solution (2.5) thus represents a Green's function for problems where contaminant is continuously injected.

There are several possible sources of error in the results $(2.5,5.1)$ and, of course, the results are only applicable for short times after injection. Major errors could be caused by diffusion to proximity of boundaries, numerical divergence of the series, or streamline curvature becoming significant.

\section{Diffusion to proximity of boundaries}

The results $(2.5,5.1)$ cannot apply in general if the solute has had sufficient time to diffuse to the proximity of boundaries, that is, for

$$
O\left(t-t_{0}\right)>L^{2} / \kappa
$$

where $L$ is a typical dimension of the domain. In the case of plane boundaries the condition $\partial C / \partial n=0$ can be met by considering dispersion in an additional image flow field and an example of such a technique was given in Section 4.

Numerical divergence of the series

The asymptotic series (2.5) should be useful for computations provided that

$$
T^{n}\left|G_{n}(\mathbf{R})\right|>T^{n+1}\left|G_{n+1}(\mathbf{R})\right|
$$

for the first few terms at least. An examination of equations $(3.1,3.2)$ shows that (5.3) is easily satisfied for

$$
T<O(\kappa / L U)
$$

(The value $\kappa / L U$ was obtained by assuming the velocity components were of order $U$ and that boundary layer effects were unimportant. Thus the order of $u_{i, j k}\left(\mathbf{r}_{0}\right)$, for example, was taken as $U / L^{2}$.) Bearing the definition (2.6) in mind, the series (2.5) might therefore become unsuitable for computation whenever

$$
O\left(t-t_{0}\right)>\kappa / U^{2}
$$

The author's experience with the computations described in Section 4 was that the estimate (5.5) is very pessimistic for the stagnation point flow. In this case there was little substantial difference in the numerical values predicted by 3 and 4 term expansions even for $t-t_{0}$ very much greater than $O\left(\kappa / U^{2}\right)$. For dispersion in flow near a point vortex and in plane Poiseuille flow, no substantial discrepancies between three and four term expansions occurred until $t-t_{0}$ was much greater than $O\left(\kappa / U^{2}\right)$ (see captions to Figs. 2 and 3 ). 


\section{Streamline curvature}

The theory described above is based on small modifications to isotropic diffusion about a point which moves along the line tangential to the streamline at the injection point. Clearly the theory will be inapplicable when streamline curvature becomes significant which may be shown to be for $t-t_{0}$ of $O(L / U)$. (Incidentally it was shown in Section 1 that diffusion dominates the dispersion mechanism for times after injection less than order (velocity gradient) ${ }^{-1}$ which is $O(L / U)$ if boundary layers are not important.)

Thus the series (2.5) will not give applicable results due to streamline curvature when $O\left(t-t_{0}\right)=L / U$. The computations described previously for the stagnation point and point vortex flows show that the results are inapplicable when

$$
t-t_{0}>c L / U
$$

where $c$ is a constant whose value is about 0.15 or 0.2 . This condition does not apply in the case of plane Poiseuille flow when the streamlines are straight.

All the above estimates need revising when considering the injection of a cloud of finite extent over a period of time as in (5.1). Suppose the contaminant cloud originally had the typical length $l$ and that the velocity field had the typical magnitude $U$ with changes occurring over a length $L$. Then the present theory, based on the modification of initial isotropic diffusion, will hold provided $\left(\kappa\left(t-t_{0}\right)\right)^{\frac{1}{2}}$ is greater than $l \times(U / L) \times\left(t-t_{0}\right)$, that is,

$$
O\left(t-t_{0}\right)<\frac{\kappa L^{2}}{U^{2} l^{2}}
$$

The importance of this restriction would depend on the character of the flow, and streamline curvature of numerical divergence may be a more likely source of errors in some flows.

It seems reasonable to expect that the series (2.5) is asymptotic to the solution of $(2.1,2.2)$ as $t \rightarrow 0$ although a proof of asymptoticity has not been attempted by the author.

In conclusion, the preceding results should be suitable to describe the initial dispersion of a cloud of solute injected into a laminar flow away from boundaries and the results should be better for flows with straight or nearly straight streamlines. The series (2.5) is applicable for only a limited time as suggested by $(5.2,5.5,5.6$, 5.7), but this time could still be important for applications. The series may be particularly relevant for the dispersion of gases in gases where the diffusivity $\kappa$ would commonly be much greater than that for dissolved solutes in fluids. For longer times, the analysis could be used to provide initial conditions for a numerical solution of equation (2.1). The possible applications to dispersion in turbulent flows such as those found in oceanography and meteorology are very restricted in 
view of the assumption of a constant diffusivity. For most turbulent flows the eddy diffusivities cannot be taken as constants; indeed, the vertical and horizontal diffusivities in geophysical situations are often taken to differ by orders of magnitude. A more comprehensive theory capable of handling some of these limitations may be attempted by the author in the future.

\section{References}

[1] M. Abramowitz and I. A. Stegun, Handbook of mathematical functions (Dover, New York, 1965).

[2] R. Aris, "On the dispersion of a solute in a fluid flowing slowly through a tube", Proc. Roy. Soc. A 235 (1956), 67-77.

[3] N. G. Barton, "The dispersion of a buoyant solute in laminar flow in a straight horizontal pipe. Part 1. Predictions from Erdogan \& Chatwin's (1967) paper”, J. Fluid Mech. 74 (1976), 81-89.

[4] N. G. Barton, "The dispersion of a buoyant solute in laminar flow in a straight horizontal pipe. Part 2. The approach to the asymptotic state", J. Fluid Mech. 74 (1976), 91-112.

[5] P. C. Chatwin, "The approach to normality of the concentration distribution of solute in solvent flowing along a straight pipe", J. Fluid Mech. 43 (1970), 321-352.

[6] P. C. Chatwin, "A calculation illustrating effects of the viscous sub-layer on longitudinal dispersion", Q. J. Mech. Appl. Math. 26 (1973), 427-439.

[7] P. C. Chatwin, "The initial dispersion of contaminant in Poiseuille flow and the smoothing of the snout", J. Fluid Mech. 77 (1976), 593-602.

[8] P. C. Chatwin, "The initial development of dispersion in straight tubes", J. Fluid Mech. 80 (1977), 33-48.

[9] J. W. Elder, "The dispersion of marked fluid in turbulent shear flow", J. Fluid Mech. 5 (1959), 544-560.

[10] M. E. Erdogan and P. C. Chatwin, "The effects of curvature and buoyancy on the laminar dispersion of solute in a horizontal tube", J. Fluid Mech. 29 (1967), 465-484.

[11] M. J. Lighthill, "Initial development of diffusion in Poiseuille flow", J. Inst. Maths Applics 2 (1965), 97-108.

[12] P. G. Saffman, "On the effect of the molecular diffusivity in turbulent diffusion", J. Fluid Mech. 8 (1960), 273-283.

[13] P. J. Sullivan, "Longitudinal dispersion within a two-dimensional turbulent shear flow", J. Fluid Mech. 49 (1971), 551-576.

[14] G. I. Taylor, "Dispersion of soluble matter in solvent flowing slowly through a tube", Proc. Roy. Soc. A 219 (1953), 186-203.

[15] G. I. Taylor, "The dispersion of matter in turbulent flow through a pipe", Proc. Roy. Soc. A 223 (1954), 446-468.

School of Mathematics

University of N.S.W.

Kensington, N.S.W. 